\title{
Multi-scale Shell Vibration Signal Analysis of Ball Mill in Grinding Process
}

\author{
Jian Tang \\ Unit 92941, PLA \\ Huludao, China \\ E-mail: tjian001@126.com
}

\author{
Zhuo Liu \\ Research Center of Automation \\ Northeastern University \\ Shenyang, China \\ E-mail: liuzhuo@mail.neu.edu.c
}

\begin{abstract}
Component decomposition and physical interpretation of shell vibration signal of ball mill in grinding process is very difficult. Aim to this problem, several multi-scales decomposition algorithms are used to analyze shell vibration signal in this paper. Empirical mode decomposition, ensemble empirical mode decomposition and Hilbert vibration decomposition algorithms decompose shell vibration signal to a series of multi-scale sub-signals. Correlation coefficients between these sub-signals and original vibration signal were calculated. Power density spectrums of different multi-scale sub-signals used also to analyze and explain the shell vibration signal. Results show that different decomposition algorithms have different advantages and shortcomings. The further research direction is that how to fuse these sub-signals and model load parameters inside the ball mill.
\end{abstract}

Keywords-multi-scale decompositon; Empirical mode decomposition; ensemble empirical decomposition; Hilbert vibration decomposition; shell vibration signal

\section{INTRODUCTION}

Mechanical devices of complex industrial process, especially rotating mechanical device in fire plant and grinding process, produce strong vibration and acoustical signals. These signals contain interesting information for diagnose mechanical devices' health statue and measure some difficulty-to-measure key process parameters, such as mill load of the ball mill in mineral grinding process. However, these signals have characteristics of nonlinearity, non-stationary and multi-scale [1]. How to effective decompose and interpret these signals using multi-scale decomposition algorithm has become a new focus recently. In this paper, the focus is how to decompose and explain the shell vibration signal of the ball mill in grinding process.

In the complex mineral grinding process, ball mills are the most important key devices in maintaining the stability, improving the grinding production rate and the products quality [2]. However, it is very difficult to maintain an optimized grinding condition for the grinding process. Accurate measure load parameters inside the ball mill can help realize grinding process operational optimization. However, as the rotating work characteristic of ball mill and complex grinding mechanism inside ball mill, it is difficulty to measure these parameters directly and calculate them in the first principal model. Thus, the shell vibration and acoustical signals have been one of the mainly indirect methods to measure these load parameters [3]. Many researches have been done on wet ball mill [4], dry ball mill [5] and SAG mill [6]. However, most of the above methods used the extract and select features from frequency spectrum obtained by traditional Fast Fourier Transform (FFT) method. They are difficult to explain and the soft sensor models cannot be interpreted clearly.

The numbers of the steel balls inside the mill are hundreds of thousands, which arranges hierarchically. The impact forces and periods of different layers' balls are different. Thus, the shell vibration signal of the ball mill has strong non-stationary and multi-scale characteristics. Empirical mode decomposition (EMD) is one effective adaptively decompose method, which can decompose the time-domain original signal into some intrinsic mode functions (IMFs) [7]. Tang et al. proposed an EMD, PSD and partial least squares (PLS) based approach to analyze the shell vibration signal and modeling load parameters of the ball mill [8]. However, the above EMD based soft sensor models have poor generalization and the prediction error even high than single-scale frequency spectrum based soft sensor model.

In order to construct multi-scale sub-signals based load parameters measuring soft sensor models with high accuracy, the first step is to obtain reliable multi-scale frequency spectrum. The original proposed EMD has many shortcomings, such as lacking a theoretical foundation, end effects, sifting stop criterion, extremum interpolation etc. Besides the shortcomings discussed above, another outstanding disadvantage of EMD method is the mode-mixing phenomenon. It is the result of intermittency. However, the newly proposed ensemble EMD (EEMD) can overcome mode-mixing problem. This method calculate the mean of an ensemble of decompositions that have different instances of noise added to the signal [9]. However, two decomposition parameters have to select for EEMD method. Thus, the adaptive ability of EMD algorithm is lost. Moreover, more computer time needs than EMD method. More recently, one of the authors proposed HVD [10]. The HVD method is theoretically based on the Hilbert-Transform (HT) presentation of the instantaneous frequency (IF) and does not involve a spline fitting and empirical algorithms. Moreover, it is natural that every inherent components of HVD may have physical and mathematical significance.

There is one common shortcoming for the above three multi-scales decomposition method. It is that only a finite small number of separated valued components can be obtained. Normally, EMD does not exceed 3 4, and HVD dose not exceed 6 8. The sub-components of EMD and EEMD methods order from high frequency to low frequency. However, these sub-components orders from 
high energy to low energy for HVD method. Thus, different sub-components contain different valued information. Comparison and analysis of these methods decomposition results can help us understand of the multiscales vibration signal.

Therefore, EMD, EEMD and HVD methods decompose the shell vibration signal of ball mill in different grinding condition to a series of multi-scale subsignals. Correlation coefficients between these sub-signals and original vibration signal were calculated. Power density spectrum of different multi-scales sub-signals used also to analyze and explain shell vibration. Results show that different sub-signals contain different information.

\section{DESCRIPTION OF SEVERAL MULTI-SCALES DECOMPOSITON ALGORITHMS}

The original EMD decomposition algorithm has been applied widely in the last decade. More modified EMD algorithms, such as ensemble EMD (EEMD), is also used successfully in different background. More recently, one of the authors proposed HVD. These algorithms can decompose multi-scale non-stationary signal to sub-signals with different interesting information.

\section{A. Emperical Decomposition Algorithm (EMD)}

The EMD method decompose original signal automatically to a serial of intrinsic mode functions (IMFs). These IMFs satisfy two conditions: (a) in a complete data set, the number of extreme and the number of zero crossings must either be equal or differ not more than by one; (b) at any point, the mean value of the envelope defined by the local maxima and the envelope defined by the local minima is zero.

The EMD algorithm is based on iteration, with the following steps:

(1)Estimation of all local extrema;

(2)Spline fitting of all local minima and maxima, ending up with two (the top and the bottom) extrema functions as the upper and the lower envelopes;

(3)Computation of the average function between the upper and the lower envelopes;

(4)Extraction of the average from the initial signal;

(5)Iteration on the residual (the sifting procedure).

\section{B. Ensemble Emperical Decomposition Algorithm (EEMD)}

The major problem of EMD method is the modemixing phenomenon. This problem results: (a) an IMF containing signals of widely disparate scales; (b) signals of similar scale residing in different IMF components. Thus, EEMD was proposed, which calculate the mean of an ensemble of decompositions that have different instances of noise added to the signal.

The EEMD algorithm is also based on an iteration, which has the following steps:

(1) Initialize the number of trials in the ensemble number $\mathrm{M}$, the amplitude of the added white noise;

(2) Add white noise to original signal and decompose the new signals with EMD;

(3) Perform M times steps (2);

(4) Calculate the ensemble mean

\section{Hilbert Vibraiton Decomposition (HVD)}

The HVD method is based on the Hilbert transform (HT) presentation of the instantaneous frequency (IF) and does not involve spline fitting and empirical algorithms. It decomposes the original signal into a sum of components with slow varying instantaneous amplitudes and frequencies. The decomposition is based on the following assumptions: (a) the underlying signal is formed by a superposition of symmetric quasi-harmonic functions; (b) the envelopes of each oscillating component differ from each other; (c) the total length of each component spans several periods of the corresponding slowest component.

Normally, every inherent components may have physical and mathematical significance. This method is also an iterative process, and every iteration step includes the following three procedures:

(1)Estimation of the IF of the largest component;

(2)Detection of the corresponding envelope of the largest component;

(3)Subtraction of the largest component from the original composition.

\section{Multi-SCALES SUB-SIGNALS ANALYSIS APPROACH OF SHELL VIBRATION SIGNAL}

It is necessary to analyze the shell vibration signal in detail. The proposed multi-scales sub-signals analysis approach consist of three modules: multi-scales signal decomposition, correlation coefficients calculation and time/frequency transform module.

\section{A. Multi-scales Signal Decomposition}

The shell vibration signal are can be represented as the summary of a series of sub-signals and a residual signal using EMD, EEMD and HVD algorithms respectively. The relationships among these sub-signals and original signal are represented as:

$$
\begin{aligned}
& \mathbf{x}=\sum_{j_{\mathrm{v}}=1}^{J_{\mathrm{VEND}}} \mathbf{x}_{\mathrm{j}_{\mathrm{END}}}^{o}+r_{\mathrm{EMD}} \\
& \mathbf{x}=\sum_{j_{\mathrm{v}}=1}^{J_{\mathrm{VEFMD}}} \mathbf{x}_{\mathrm{jEEMD}_{\mathrm{EEM}}}^{o}+r_{\mathrm{EEMD}} \\
& \mathbf{x}=\sum_{j_{\mathrm{V}}=1}^{J_{\mathrm{VHVD}}} \mathbf{x}_{\mathrm{j}_{\mathrm{HVD}}}^{o}+r_{\mathrm{HVD}}
\end{aligned}
$$

where, $\mathbf{x}$ represent the original shell vibration signal; $\mathbf{x}_{\mathrm{j}_{\mathrm{EMD}}^{o}}^{o}, \mathbf{x}_{\mathrm{j}_{\mathrm{EEMD}}}^{o}$ and $\mathbf{x}_{\mathrm{j}_{\mathrm{HVD}}}^{o}$ represent the $j_{\mathrm{EMD}}$ th , $j_{\text {EEMD }}$ th and $j_{\text {HVD }}$ th multi-scale sub-signals using EMD, EEMD and HVD algorithms respectively; $r_{\mathrm{EMD}}$, $r_{\mathrm{EEMD}}$ and $r_{\mathrm{HVD}}$ represent residual signals of shell vibration decomposition; $J_{\mathrm{EMD}}, J_{\mathrm{EEMD}}$ and $J_{\mathrm{HVD}}$ represent number of sub-signals.

\section{B. Correlation Coefficients Calculation}

It is important to judge and select sub-signals that have strong correlation with original signal. Studies show that only a finite small number of separated valued components can be obtained with multi-scale decomposition algorithm. The following equation can calculate the correlation coefficients between different sub-signals and original signal: 


$$
\begin{aligned}
& r_{j \mathrm{EMD}}=\frac{\sum(\mathbf{x}-\overline{\mathbf{x}})\left(\mathbf{x}_{\mathrm{E} \text { EMD }}^{o}-\overline{\mathbf{x}}_{\mathrm{E} \text { EMD }}^{o}\right)}{\sum(\mathbf{x}-\overline{\mathbf{x}})^{2} \sum\left(\mathbf{x}_{\mathrm{jEMD}}^{o}-\overline{\mathbf{x}}_{\mathrm{jEMD}}^{o}\right)^{2}} \\
& r_{j_{E E M D}}=\frac{\sum(\mathbf{x}-\overline{\mathbf{x}})\left(\mathbf{x}_{\text {jEEMD }}^{o}-\overline{\mathbf{x}}_{\text {jEEND }}^{o}\right)}{\sum(\mathbf{x}-\overline{\mathbf{x}})^{2} \sum\left(\mathbf{x}_{\text {jEEM }}^{o}-\overline{\mathbf{x}}_{\text {jEEMD }}^{o}\right)^{2}} \\
& r_{j_{\mathrm{HVD}}}=\frac{\sum(\mathbf{x}-\overline{\mathbf{x}})\left(\mathbf{x}_{\mathrm{j}_{\mathrm{HVD}}^{o}}-\overline{\mathbf{x}}_{\mathrm{jHVD}_{\mathrm{HV}}^{o}}^{o}\right)}{\left.\sum(\mathbf{x}-\overline{\mathbf{x}})^{2} \sum\left(\mathbf{x}_{\mathrm{HVD}}^{o}-\overline{\mathbf{x}}_{\mathrm{HHV}}^{o}\right)\right)^{2}}
\end{aligned}
$$

where, $r_{j_{E \mathrm{MD}}}, r_{j_{E E \mathrm{MD}}}$ and $r_{j_{\mathrm{HVD}}}$ represent correlation coefficient between original signal and the $j_{\mathrm{EMD}}$ th , $j_{\text {EEMD }}$ th and $j_{\mathrm{HVD}}$ th sub-signal using EMD, EEMD and HVD algorithm decomposed respectively.

At the same time, the hypothesis of no correlation values $p_{j_{E \mathrm{MD}}}, p_{j_{E E \mathrm{MD}}}$ and $p_{j_{\mathrm{HVD}}}$ are also calculated. Given threshold $p_{\text {threshold }}$, the following criterion is used to selecting shell vibration sub-signals:

$$
\xi_{j \mathrm{sel}}=\left\{\begin{array}{lll}
1 & \text { if } & r_{j_{\mathrm{EMD}}} \mathrm{OR} r_{j_{\mathrm{EEMI}}} \mathrm{OR} r_{j_{\mathrm{HMD}}} \geq p_{\text {threshold }} \\
0 & \text { else } & r_{j_{\mathrm{EMD}}} \mathrm{OR} r_{j_{\mathrm{EEMD}}} \mathrm{OR} r_{j_{\mathrm{HMD}}}<p_{\text {threshold }}
\end{array}\right.
$$

Using the above criterion, the sub-signals with $\xi_{j \text { sel }}=1$ are selected as effective components of the original signal. Denoted the selected sub-signals as $\mathbf{x}_{\substack{\text { jel } \\ \text { jel }}}, \mathbf{x}_{\text {jel }}^{\text {jel }}$ and $\mathbf{x}_{\text {jel }}$, and their numbers are $J_{\mathrm{EMD}}^{\mathrm{sel}}, J_{\mathrm{EEMD}}^{\mathrm{sel}}$ and $J_{\mathrm{HVD}}^{\mathrm{sel}}$ respectively.

\section{Time/Frequency Transform}

As the impact force on any point of the ball mill shell is different at different time during the mill rotate period. The data length using for time/frequency transform is at least one mill rotate period. Standard Welch's method is used to calculate the power spectral density (PSD). The following equation can represent the multi-scales frequency spectrum obtain process:

$$
\begin{aligned}
& \mathbf{x}_{j \mathrm{EMD}} \stackrel{\mathrm{FFT}}{\longrightarrow} \mathbf{z}_{j_{\mathrm{EMM}}^{\mathrm{sel}}} \\
& \mathbf{x}_{j \mathrm{sel}} \stackrel{\mathrm{FFT}}{\longrightarrow} \mathbf{z}_{j_{\mathrm{EEEMD}}^{\mathrm{sel}}} \\
& \mathbf{x}_{j \mathrm{HVl}} \stackrel{\mathrm{FFT}}{\longrightarrow} \mathbf{z}_{{ }_{j \mathrm{HVD}}^{\mathrm{sel}}}
\end{aligned}
$$

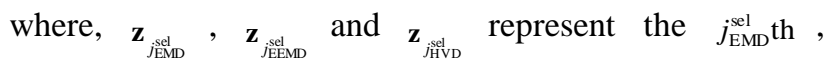
$j_{\mathrm{EEMD}}^{\text {sel }}$ th and $j_{\mathrm{HVD}}^{\mathrm{sel}}$ th sub-signal frequency spectrum using EMD, EEMD and HVD method respectively.

\section{APPLICATION RESUlTS}

The experiments are performed on a laboratory scale ball mill (XMQL-420×450). The copper ore was crushed to about less than $6 \mathrm{~mm}$ before used. The diameters of the steel balls are 30,20 and $15 \mathrm{~mm}$ respectively. The vibration and acoustical signals were picked up by accelerometer located on the middle of the mill shell. The detail is shown in [4], which are omitted in here.

In this paper, only shell vibration signals of four different grinding conditions, such as zero load, ball load, dry mill and wet mill, are decomposed and analyzed. The differences are: (1) There is not any load in zero grinding condition; (2) There is $40 \mathrm{~kg}$ steel balls in ball load grinding condition; (3) There is $40 \mathrm{~kg}$ steel balls and $30 \mathrm{~kg}$ mineral ores in dry mill grinding condition; (4) There is
$40 \mathrm{~kg}$ steel balls, $30 \mathrm{~kg}$ mineral ores and $10 \mathrm{~kg}$ water in wet mill grinding condition.

\section{A. Original Shell Vibration Signals}

The original shell vibration signals of two mill rotate periods at different grinding condition are shown as Fig.1.
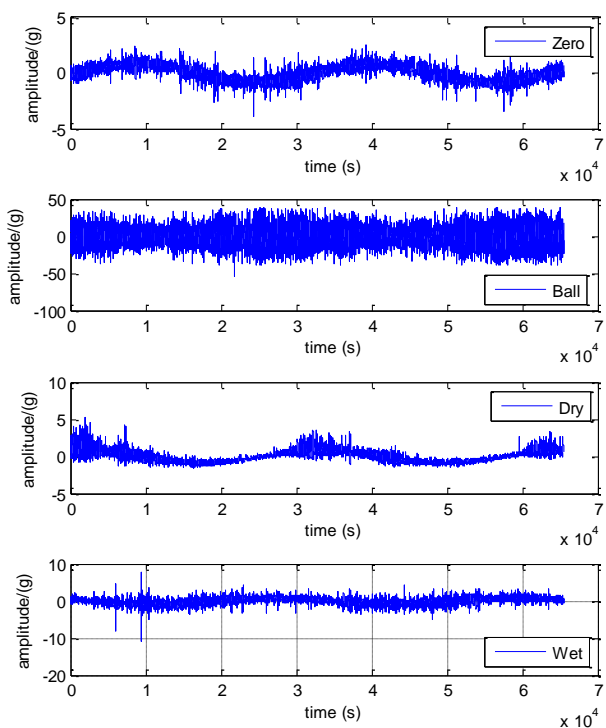

Figure 1. Original shell vibration signals of two mill rotate periods

Fig.1 shows that the laboratory scale ball mill has itself vibration with amplitude $5 \mathrm{~g}$, and the dry and wet mill can decrease the vibration amplitude from $50 \mathrm{~g}$ to $10 \mathrm{~g}$. The components of shell vibration signals at different grinding condition are different.

\section{B. Multi-scale Decompositon Results}

The following decompose parameters are used: EMD with the default, EEMD with the ensemble number 10 and white noise amplitude 0.1, and HVD with subcomponent 15 and others default. The multi-scale decomposition results of the former five sub-signals are shown as Fig. 2 Fig. 4.

Fig. 2 Fig. 4 show that: (1) Sub-signals of EEMD has higher amplitude than that of EMD and HVD; (2) The first sub-signals of EMD and HVD are two period signals zero load, which different from EMD; (3) Except dry mill, most of the sub-signals different cannot be distinguish clearly. Thus, correlation coefficients based detailed analysis is necessary.

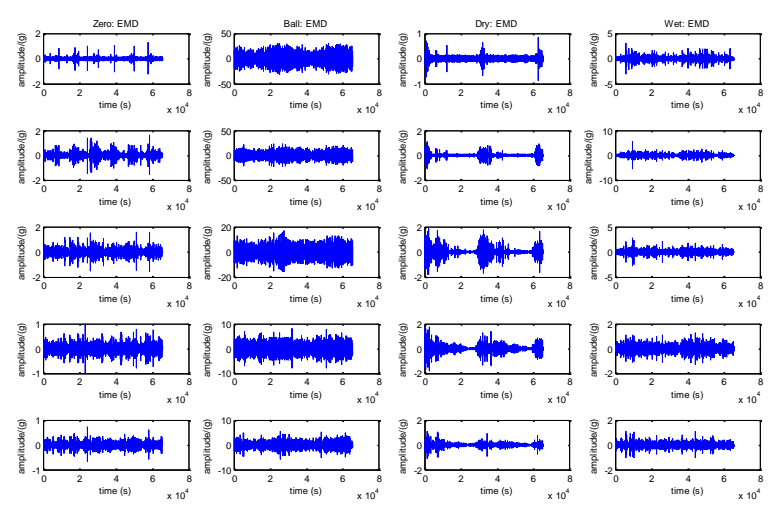

Figure 2. Decomposition results of former five sub-signals with EMD 


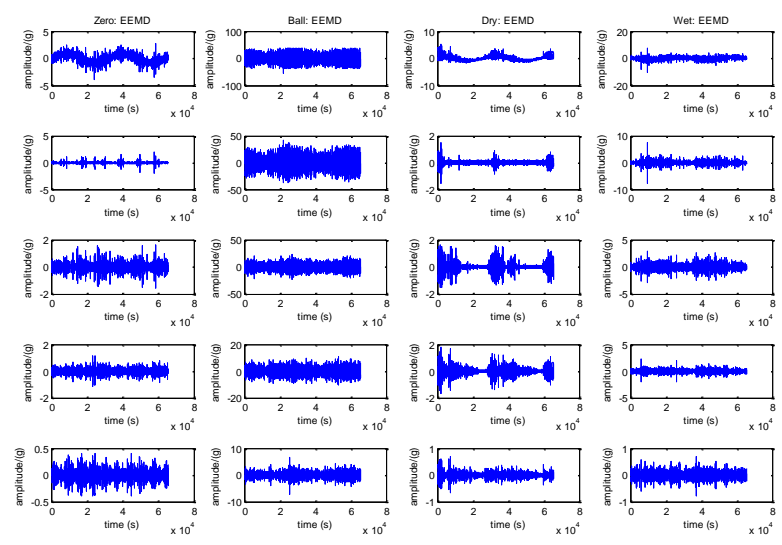

Figure 3. Decomposition results of former five sub-signals with EEMD

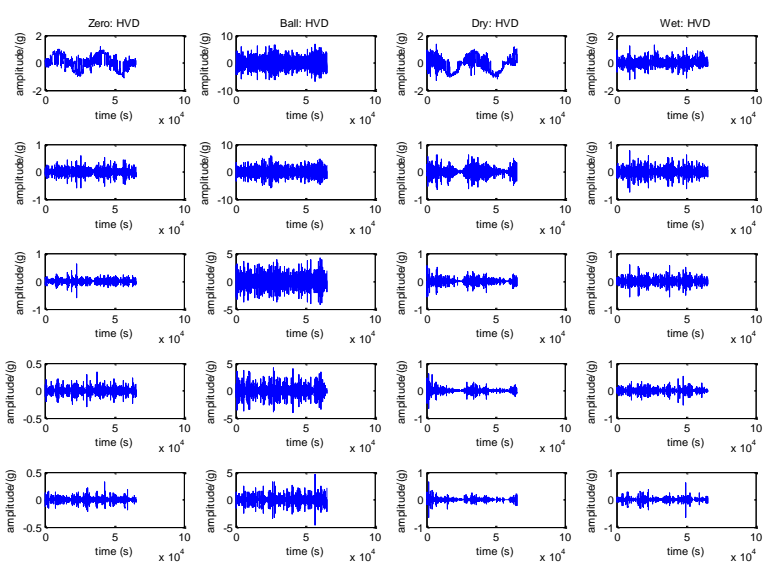

Figure 4. Decomposition results of former five sub-signals with HVD

\section{Correlation Analysis Results}

Using default decomposition parameters of EMD, there are 14 sub-signals in zero grinding condition, and the last one is residual signal. For uniform, we only analyze the form 13 sub-signals at different grinding condition. Correlation coefficients statistical results are shown as Table 1 Table 4.

Table 1 Statistical results of correlation coefficients for zero load

\begin{tabular}{|c|c|c|c|c|c|c|c|}
\hline \multirow{3}{*}{ Number } & \multicolumn{6}{|c|}{ Decompose algorithm and results } & \multirow{3}{*}{ Note } \\
\hline & \multicolumn{2}{|c|}{ EMD } & \multicolumn{2}{|c|}{ EEMD } & \multicolumn{2}{|c|}{ HVD } & \\
\hline & $\mathrm{r}$ & $\mathrm{p}$ & $\mathrm{t}$ & $\mathrm{p}$ & $\mathrm{r}$ & $\mathrm{p}$ & \\
\hline 1 & 0.1316 & 0 & 1 & 0 & 0.7037 & 0 & \\
\hline 2 & 0.2423 & 0 & 0.1954 & 0 & 0.2073 & 0 & \\
\hline 3 & 0.3205 & 0 & 0.3718 & 0 & 0.1590 & 0 & \\
\hline 4 & 0.2382 & 0 & 0.3478 & 0 & 0.1333 & 0 & \\
\hline 5 & 0.1446 & 0 & 0.1861 & 0 & 0.1088 & 0 & \\
\hline 6 & 0.0592 & 0 & 0.0721 & 0 & 0.0888 & 0 & \\
\hline 7 & 0.0285 & 0.5442 & 0.0776 & 0 & 0.0786 & 0 & \\
\hline 8 & 0.0684 & 0 & 0.1102 & 0 & 0.0666 & 0 & \\
\hline 9 & 0.0908 & 0 & 0.0916 & 0 & 0.0617 & 0 & \\
\hline 10 & 0.0602 & 0 & 0.0479 & 0 & 0.0658 & 0 & \\
\hline 11 & 0.0423 & 0 & 0.2647 & 0 & 0.0593 & 0 & \\
\hline 12 & 0.0840 & 0 & 0.8526 & 0 & 0.0533 & 0 & \\
\hline 13 & 0.8563 & 0 & 0.4514 & 0 & 0.0563 & 0 & \\
\hline
\end{tabular}

Table 2 Statistical results of correlation coefficients for ball load

\begin{tabular}{|c|c|c|c|c|c|c|c|}
\hline \multirow{3}{*}{$\begin{array}{l}\mathrm{Nu} \\
\text { mbe }\end{array}$} & \multicolumn{6}{|c|}{ Decompose algorithm and results } & \multirow{3}{*}{ Note } \\
\hline & \multicolumn{2}{|c|}{ EMD } & \multicolumn{2}{|c|}{ EEMD } & \multicolumn{2}{|c|}{ HVD } & \\
\hline & $\mathrm{r}$ & $\mathrm{p}$ & $\mathrm{r}$ & $\mathrm{p}$ & $\mathrm{r}$ & $\mathrm{p}$ & \\
\hline 1 & 0.7735 & 0 & 1 & 0 & 0.2082 & 0 & \\
\hline 2 & 0.5024 & 0 & 0.1954 & 0 & 0.1770 & 0 & \\
\hline 3 & 0.3369 & 0 & 0.3718 & 0 & 0.1517 & 0 & \\
\hline 4 & 0.1502 & 0 & 0.3478 & 0 & 0.1323 & 0 & \\
\hline 5 & 0.0613 & 0 & 0.1861 & 0 & 0.1088 & 0 & \\
\hline 6 & 0.0276 & 0 & 0.0721 & 0 & 0.0920 & 0 & \\
\hline 7 & 0.0142 & 0.5442 & 0.0776 & 0 & 0.0850 & 0 & \\
\hline 8 & 0.0089 & 0.0012 & 0.1102 & 0 & 0.0816 & 0 & \\
\hline 9 & 0.0066 & 0.0158 & 0.0916 & 0 & 0.0683 & 0 & \\
\hline 10 & 0.0087 & 0.0015 & 0.0479 & 0.0167 & 0.0623 & 0 & \\
\hline 11 & 0.0059 & 0.0304 & 0.2647 & 0.3507 & 0.0524 & 0 & \\
\hline 12 & 0.0021 & 0.4314 & 0.8526 & 0.0262 & 0.0485 & 0 & \\
\hline 13 & 0.0029 & 0.2796 & 0.4514 & 0 & 0.0534 & 0 & \\
\hline
\end{tabular}

Table 3 Statistical results of correlation coefficients for dry mill

\begin{tabular}{|c|c|c|c|c|c|c|c|}
\hline \multirow{3}{*}{ Number } & \multicolumn{6}{|c|}{ Decompose algorithm and results } & \multirow{3}{*}{ Note } \\
\hline & \multicolumn{2}{|c|}{ EMD } & \multicolumn{2}{|c|}{ EEMD } & \multicolumn{2}{|c|}{ HVD } & \\
\hline & $\mathrm{r}$ & $\mathrm{p}$ & $\mathrm{r}$ & $\mathrm{p}$ & $\mathrm{r}$ & $\mathrm{p}$ & \\
\hline 1 & 0.0819 & 0 & 1 & 0 & 0.7930 & 0 & \\
\hline 2 & 0.1248 & 0 & 0.1389 & 0 & 0.2077 & 0 & \\
\hline 3 & 0.2799 & 0 & 0.3025 & 0 & 0.1583 & 0 & \\
\hline 4 & 0.2831 & 0 & 0.3687 & 0 & 0.1448 & 0 & \\
\hline 5 & 0.1601 & 0 & 0.1652 & 0 & 0.1052 & 0 & \\
\hline 6 & 0.0850 & 0 & 0.0866 & 0 & 0.1033 & 0 & \\
\hline 7 & 0.0474 & 0 & 0.1120 & 0 & 0.0813 & 0 & \\
\hline 8 & 0.0765 & 0 & 0.1186 & 0 & 0.0718 & 0 & \\
\hline 9 & 0.0878 & 0 & 0.0955 & 0 & 0.0621 & 0 & \\
\hline 10 & 0.1062 & 0 & 0.0358 & 0 & 0.0582 & 0 & \\
\hline 11 & 0.0297 & 0 & 0.0318 & 0 & 0.0584 & 0 & \\
\hline 12 & 0.0217 & 0 & 0.8503 & 0 & 0.0571 & 0 & \\
\hline 13 & 0.0231 & 0 & 0.0515 & 0 & 0.0463 & 0 & \\
\hline
\end{tabular}

Table 3 Statistical results of correlation coefficients for wet mill

\begin{tabular}{|c|c|c|c|c|c|c|c|}
\hline \multirow{3}{*}{$\begin{array}{l}\mathrm{Nu} \\
\text { mbe }\end{array}$} & \multicolumn{6}{|c|}{ Decompose algorithm and results } & \multirow{3}{*}{ Note } \\
\hline & \multicolumn{2}{|c|}{ EMD } & \multicolumn{2}{|c|}{ EEMD } & \multicolumn{2}{|c|}{ HVD } & \\
\hline & $\mathrm{r}$ & $\mathrm{p}$ & $\mathrm{r}$ & $\mathrm{p}$ & $\mathrm{r}$ & $\mathrm{p}$ & \\
\hline 1 & 0.3031 & 0 & 1 & 0 & 0.3484 & 0 & \\
\hline 2 & 0.4542 & 0 & 0.4627 & 0 & 0.2311 & 0 & \\
\hline 3 & 0.3962 & 0 & 0.5273 & 0 & 0.1731 & 0 & \\
\hline 4 & 0.2890 & 0 & 0.3663 & 0 & 0.1449 & 0 & \\
\hline 5 & 0.1706 & 0 & 0.1844 & 0 & 0.1196 & 0 & \\
\hline 6 & 0.0952 & 0 & 0.0898 & 0 & 0.1043 & 0 & \\
\hline 7 & 0.0401 & 0.5442 & 0.0694 & 0 & 0.0954 & 0 & \\
\hline 8 & 0.0306 & 0 & 0.0915 & 0 & 0.0807 & 0 & \\
\hline 9 & 0.0520 & 0 & 0.0700 & 0 & 0.0863 & 0 & \\
\hline 10 & 0.0594 & 0 & 0.0402 & 0 & 0.0685 & 0 & \\
\hline 11 & 0.0405 & 0 & 0.2123 & 0 & 0.0661 & 0 & \\
\hline 12 & 0.0344 & 0 & 0.6598 & 0 & 0.0607 & 0 & \\
\hline 13 & 0.6416 & 0 & 0.1759 & 0 & 0.0619 & 0 & \\
\hline
\end{tabular}

Table 1 Table 4 shows that: (1) Sub-signals of HVD are all correlate with the original signal, which different from that of EMD and EEMD; (2) The largest correlation coefficient value is the first sub-signal of EEMD and the second correlate sub-signal is the first sub-signal of EEMD, which show EEMD and HVD have better sub- 
signal decomposition result s than EMD; (3) Ball load grinding condition result different from others; (4) Under different grinding condition, sub-signals correlation are different for EMD and EEMD algorithm. Thus, HVD and EEMD are more effective. Combine with frequency spectrum, more information can be obtained.

\section{Time-Frequency Transform results}

The time domain sub-signals of different grinding condition using EMD, EEMD and HVD method are transform into frequency domain using the Standard Welch's method with same parameters as literature [4]. The multi-scale frequency spectrum results of the former five sub-signals are shown as Fig. 5 Fig. 7.
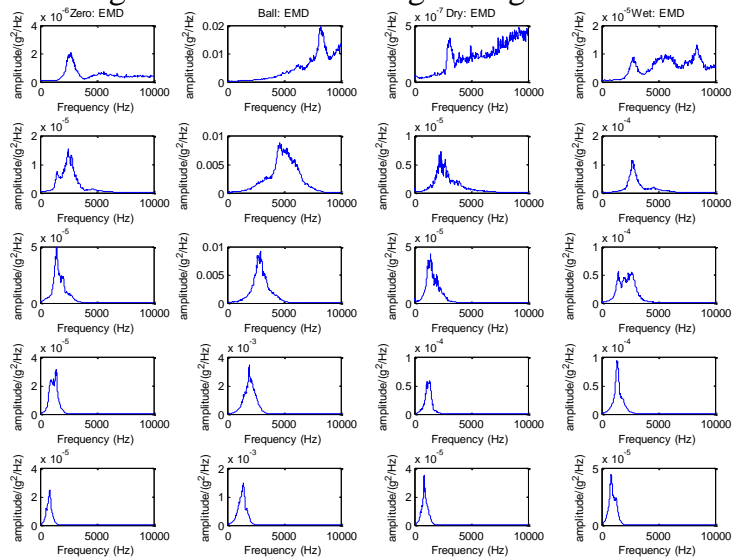

Figure 5. Frequency spectrum of the former five sub-signals with EMD
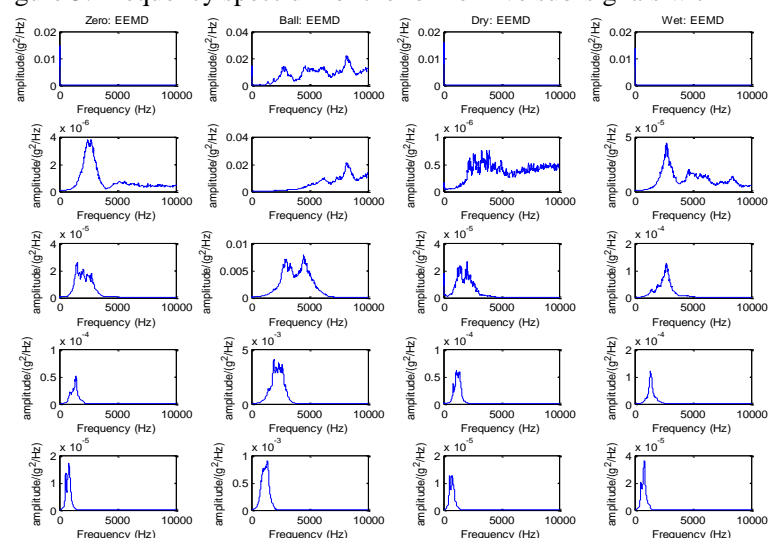

Figure 6. Frequency spectrum of the former five sub-signals with EEMD
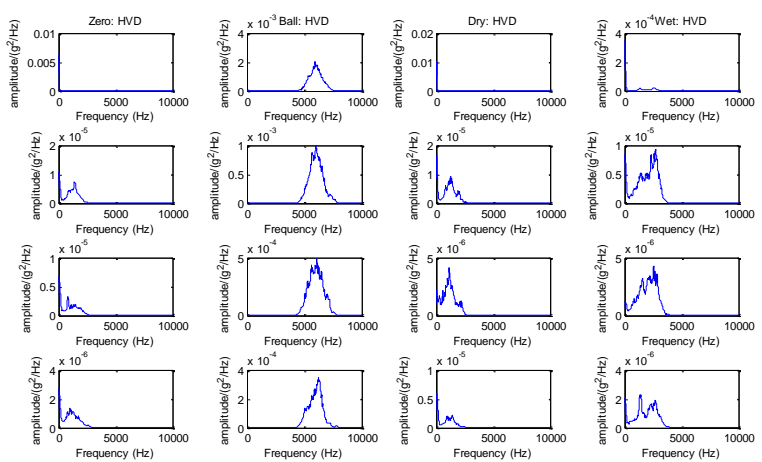

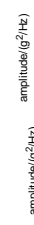
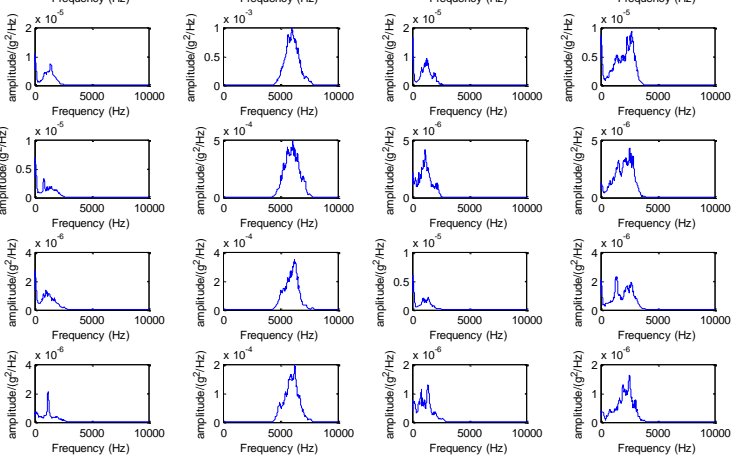

Figure 7. Frequency spectrum of the former five sub-signals with HVD
Fig. 5 Fig. 7 shows that: (1) Frequency ranges of EMD sub-signals decrease gradually, and there is evident mix-modeling in the first sub-signal; (2) Except ball load grinding condition, frequencies of the first sub-signal of EEMD and HVD are all $60 \mathrm{~Hz}$; (3) Frequency ranges of HVD sub-signals does not decrease gradually from the second sub-signal to the last one; (4) Some sub-signals of EEMD exit still multi-modeling; (5) Ball load grinding condition has very different frequency spectrum.

All the above results show that multi-scale frequency spectrums of different decompose algorithms contains different valued information.

\section{CONCLUSIONS}

Three multi-scales decomposition algorithm, such as empirical model decomposition, ensemble empirical model decomposition and Hilbert vibration decomposition, are used to decompose shell vibration signal under zero, ball load, dry mill and wet mill grind conditions. We obtain the following conclusions: (1) EEMD can decompose the most correlate sub-signals to original vibration signal; (2) The former three sub-signals of HVD have closely relationship with the original signals; (3) The correlation between sub-signals and original signal under different grinding condition are different; (4) Only the former $4 \sim 5$ frequency spectrum may have more contribution for modeling load parameters of ball mill.

\section{REFERENCES}

[1] Y.G., Lei, J., Lin, Z.J., He, and M.J., Zuo, "A review on empirical model decomposition in fault diagnosis of rotating machinery," Mechanical System and Signal Processing, vol. 35, pp. 108-126, 2013.

[2] P, Zhou, T. Y., Chai and H.,Wang, "Intelligent optimal-setting control for grinding circuits of mineral processing," IEEE Transactions on Automation Science and Engineering, vol. 6, pp. 730-743, 2009.

[3] J. Tang, L.J. Zhao, H. Yue and T.Y. Chai, "Present status and future developments of detection method for mill load," Control Engineering of China, vol. 17, pp. 565-570, 2010.

[4] J. Tang, L.J. Zhao, J. W. Zhou, H. Yue and T.Y. Chai, "Experimental analysis of wet mill load based on vibration signals of laboratory-scale ball mill shell," Minerals Engineering, vol. 23, pp. 720-730, 2010.

[5] P. Huang, M.P. Jia and B. L. Zhong, "Investigation on measuring the fill level of an industrial ball mill based on the vibration characteristics of the mill shell," Minerals Engineering, vol. 14, pp. 1200-1208, 2009.

[6] S. P., Das, D. P., Das, S. K., Behera, amd B. K., Mishra, "Interpretation of mill vibration signal via wireless sensing," Minerals Engineering, vol. 24, pp. 245-251. 2011.

[7] N. E. Huang, Z. Shen, andS. R Long, "The empirical mode decomposition and the Hilbert spectrum for non-linear and non stationary time series analysis," Proc. Royal Soc. London A, vol. 454, pp. 903-995, 1998.

[8] J. Tang, L.J. Zhao, J. Jia, T.Y. Chai, and W. Yu, "Selective ensemble modeling parameters of mill load based on shell vibration signal," Lecture Notes in Computer Science, vol. 7367 pp. 489-497, 2012.

[9] Z. H. Wu, and N. E., Huang, "Ensemble empirical mode decomposition for high frequency ECG noise reduction," Advances in Adaptive Data Analysis, vol. 55, pp. 193-201, 2009.

[10] M. Feldmann, Hilbert Transform Applications in Mechanical Vibration. Hoboken, N.J.: Wiley, 1 edition, 2011. 